\section{BMJ Open \\ Respiratory \\ Research}

\title{
British Thoracic Society Guideline for oxygen use in adults in healthcare and emergency settings
}

\author{
B R O'Driscoll, L S Howard, J Earis, V Mak, on behalf of the BTS Emergency \\ Oxygen Guideline Development Group
}

To cite: O'Driscoll BR, Howard LS, Earis J, et al. British Thoracic Society Guideline for oxygen use in adults in healthcare and emergency settings. BMJ Open Resp Res 2017;4: e000170. doi:10.1136/ bmjresp-2016-000170

- Additional material is available. To view please visit the journal (http://dx.doi.org/ 10.1136/bmjresp-2016000170)

The concise version of the BTS Guideline should be read in conjunction with the full version: https://www.britthoracic.org.uk/guidelinesand-quality-standards/ emergency-oxygen-use-inadult-patients-guideline/ This guideline replaces the 2008 British Thoracic Society guideline for emergency oxygen use.

Accepted 17 November 2017

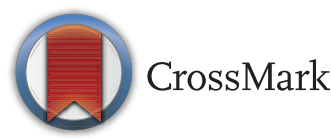

Department of Respiratory Medicine, Salford Royal Foundation NHS Trust, Salford, UK

Correspondence to Dr BR O'Driscoll; ronan.o'driscoll@srft.nhs.uk
The full Guideline for oxygen use in adults in healthcare and emergency settings, published in Thorax ${ }^{1}$ provides an update to the 2008 BTS Emergency oxygen guideline. ${ }^{2}$ The following is a summary of the recommendations and good practice points. The sections noted to within this summary refer to the full guideline sections.

\section{EXECUTIVE SUIMIMARY}

Philosophy of the guideline

$\checkmark$ Oxygen is a treatment for hypoxaemia, not breathlessness. Oxygen has not been proven to have any consistent effect on the sensation of breathlessness in nonhypoxaemic patients.

$\checkmark$ The essence of this guideline can be summarised simply as a requirement for oxygen to be prescribed according to a target saturation range and for those who administer oxygen therapy to monitor the patient and keep within the target saturation range.

$\checkmark$ The guideline recommends aiming to achieve normal or near-normal oxygen saturation for all acutely ill patients apart from those at risk of hypercapnic respiratory failure or those receiving terminal palliative care.

1. Assessing patients

O For critically ill patients, high concentration oxygen should be administered immediately (table 1 and figure 1) and this should be recorded afterwards in the patient's health record.

$\bigcirc$ Clinicians must bear in mind that supplemental oxygen is given to improve oxygenation, but it does not treat the underlying causes of hypoxaemia which must be diagnosed and treated as a matter of urgency.

$\bigcirc$ The oxygen saturation should be checked by pulse oximetry in all breathless and acutely ill patients, "the fifth vital sign' (supplemented by blood gases when necessary), and the inspired oxygen concentration should be recorded on the observation chart with the oximetry result. (The other vital signs are pulse rate, blood pressure, temperature and respiratory rate).

O Pulse oximetry must be available in all locations where emergency oxygen is used. Clinical assessment is recommended if the saturation falls by $\geq 3 \%$ or below the target range for the patient.

O All critically ill patients outside of a critical care area (e.g. intensive care unit (ICU), high dependency unit (HDU), respiratory HDU), should be assessed and monitored using a recognised physiological track and trigger system such as the National Early Warning Score (NEWS).

2. Target Oxygen prescription

O Oxygen should be prescribed to achieve a target saturation of $94-98 \%$ for most acutely ill patients or $88-92 \%$ or patient-specific target range for those at risk of hypercapnic respiratory failure (tables 1-4).

O Best practice is to prescribe a target range for all hospital patients at the time of admission so that appropriate oxygen therapy can be started in the event of unexpected clinical deterioration with hypoxaemia and also to ensure that the oximetry section of the early warning score (EWS) can be scored appropriately.

O The target saturation should be written (or ringed) on the drug chart or entered in an electronic prescribing system (guidance on figure 1).

3. Oxygen administration

O Oxygen should be administered by staff who are trained in oxygen administration. 
Table 1 Critical illnesses requiring high levels of supplemental oxygen (section 8.10)

The initial oxygen therapy is a reservoir mask at $15 \mathrm{l} / \mathrm{min}$ pending the availability of reliable oximetry readings. For patients with spontaneous circulation and a reliable oximetry reading, it may quickly become possible to reduce the oxygen dose whilst maintaining a target saturation range of $94-98 \%$.

If oximetry is unavailable, continue to use a reservoir mask until definitive treatment is available. Patients with COPD and other risk factors for hypercapnia who develop critical illness should have the same initial target saturations as other critically ill patients pending the results of blood gas results after which these patients may need controlled oxygen therapy with target range $88-92 \%$ or supported ventilation if there is severe hypoxaemia and/or hypercapnia with respiratory acidosis.

\begin{tabular}{ll}
\hline Cardiac arrest or resuscitation & Additional comments \\
\hline & Refer to resuscitation guidelines for choice of delivery \\
& device during active resuscitation. \\
& Give highest possible inspired oxygen concentration \\
during CPR until spontaneous circulation has been & restored.
\end{tabular}

Shock, sepsis, major trauma, drowning,

Also give specific treatment for the underlying anaphylaxis, major pulmonary haemorrhage, condition. Recommendations status epilepticus

Major head injury

Carbon monoxide poisoning
Early tracheal intubation and ventilation if comatose.

Give as much oxygen as possible using a bag-valve mask or reservoir mask. Check carboxyhaemoglobin levels.

A normal or high oximetry reading should be disregarded because saturation monitors cannot differentiate between carboxyhaemoglobin and oxyhaemoglobin, owing to their similar absorbances. The blood gas $\mathrm{PO}_{2}$ will also be normal in these cases (despite the presence of tissue hypoxia).

Recommendation E1

These staff should use appropriate devices and flow rates in order to achieve the target saturation range (figure 2).

$\bigcirc$ Staff should be trained in the use of a range of different oxygen delivery devices to ensure oxygen is delivered safely.

4. Monitoring and maintenance of target saturation

$\bigcirc$ Oxygen saturation and delivery system (including flow rate) should be recorded on the patient's monitoring chart.

Oxygen delivery devices and flow rates should be adjusted to keep the oxygen saturation in the target range. Prompt clinical assessment is required if oxygen therapy needs to be initiated or increased due to a falling saturation level.

$\bigcirc$ Oxygen should be prescribed and a signature should be entered on the drug chart on each drug round.

5. Weaning and discontinuation of oxygen therapy

$\bigcirc$ Oxygen should be reduced in stable patients with satisfactory oxygen saturation.

$\bigcirc$ Oxygen should be discontinued once the patient can maintain saturation within or above the target range breathing air but the prescription for a target range should be left in place in case of future deterioration and to guide early warning scores (EWS/NEWS).

\section{KEY CHANGES SINCE THE FIRST EDITION OF THIS GUIDELINE PUBLISHED IN 2008 \\ Methodology}

The evidence review methodology has changed from NICE methodology to the BTS NICE accredited guideline production process which is based on the Scottish Intercollegiate Guideline Network (SIGN) methodology and adheres to AGREE methodology (see section 1).

\section{Evidence levels and grade of recommendation}

These are now in SIGN format (see section 1 and tables 6 and 7).

\section{Evidence base}

The evidence base for the guideline has been updated to August 2013 (and extended to mid-2016 for key references). None of the 2008 recommendations have been challenged by new evidence, but many of the existing recommendations are supported by new information. There have been many observational studies but few randomised trials directly relevant to the guideline since 2008.

\section{The remit of the guideline has been extended}

The new guideline covers not just emergency oxygen use but most oxygen use in healthcare settings. It also covers short-term oxygen use by healthcare workers 


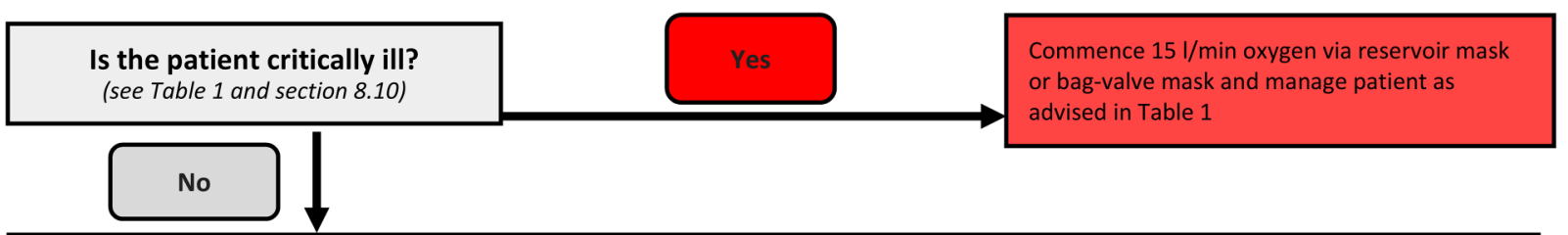

Is this patient at risk of hypercapnic respiratory failure? (Type 2 Respiratory Failure)

The main risk factor is severe or moderate COPD (Especially with previous Respiratory Failure or on Long Term Oxygen).

Other patients at risk include people with severe chest wall or spinal disease (e.g. kypho-scoliosis), neuro-muscular disease, severe obesity,

cystic fibrosis, bronchiectasis or previously un-recognised COPD

Narcotic/ sedative overdose not covered by this algorithm- see Table 4 and section 8.13 .5

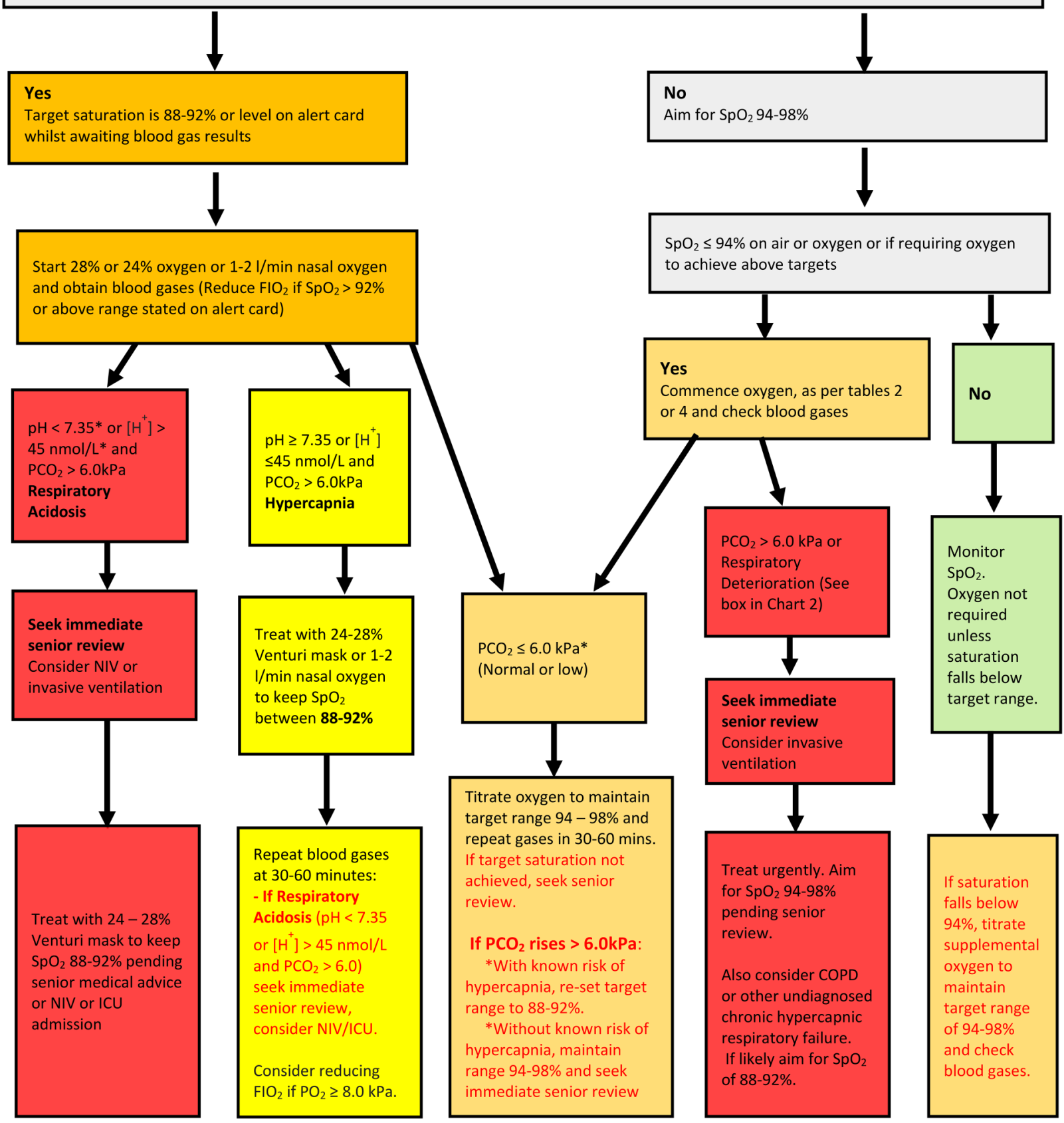

Any increase in FIO2 must be followed by repeat blood gases in 1 hour (or sooner if conscious level deteriorates)

* If $\mathrm{pH}$ is $<7.35\left(\left[\mathrm{H}^{+}\right]>45 \mathrm{nmol} / \mathrm{L}\right)$ with normal or low PCO2, investigate and treat for metabolic acidosis and keep SpO2 94-98\%

Figure 1 Oxygen prescription guidance for acutely hypoxaemic patients in hospital. COPD, chronic obstructive pulmonary disease; ICU, intensive care unit; NIV, non-invasive ventilation; $\mathrm{PO}_{2}$, oxygen tension; $\mathrm{PCO}_{2}$, arterial or arteriolised carbon dioxide tension; $\mathrm{SpO}_{2}$, arterial oxygen saturation measured by pulse oximetry. 
Table 2 Serious illnesses requiring moderate levels of supplemental oxygen if the patient is hypoxaemic (section 8.11)

The initial oxygen therapy is nasal cannulae at 2-6 L/min (preferably) or simple face mask at 5-10 L/min unless stated otherwise.

For patients not at risk of hypercapnic respiratory failure who have saturation below $85 \%$, treatment should be started with a reservoir mask at $15 \mathrm{~L} / \mathrm{min}$ and the recommended initial oxygen saturation target range is $94-98 \%$. If oximetry is not available, give oxygen as above until oximetry or blood gas results are available. Change to reservoir mask if the desired saturation range cannot be maintained with nasal cannulae or simple face mask (and ensure that the patient is assessed by senior medical staff).

If these patients have coexisting COPD or other risk factors for hypercapnic respiratory failure, aim at a saturation of 88-92\% pending blood gas results but adjust to $94-98 \%$ if the $\mathrm{PCO}_{2}$ is normal (unless there is a history of previous hypercapnic respiratory failure requiring NIV or IMV) and recheck blood gases after 30-60 min, see table 4.

\begin{tabular}{lll}
\hline & Additional comments & Recommendations \\
\hline $\begin{array}{l}\text { Acute hypoxaemia (cause not yet } \\
\text { diagnosed) }\end{array}$ & Reservoir mask at $15 \mathrm{~L} / \mathrm{min}$ if initial $\mathrm{SpO}_{2}$ below 85\%, & Recommendations \\
& otherwise nasal cannulae or simple face mask. & $\mathrm{D} 1-\mathrm{D} 3$
\end{tabular}

Acute asthma Patients requiring reservoir mask therapy need urgent clinical assessment by senior staff.

Pneumonia

Recommendations

Lung cancer

Deterioration of lung fibrosis or other interstitial lung disease

Pneumothorax

Reservoir mask at $15 \mathrm{~L} / \mathrm{min}$ if initial $\mathrm{SpO}_{2}$ below $85 \%$, F1-F3 otherwise nasal cannulae or simple face mask

Needs aspiration or drainage if the patient is hypoxaemic. Most Recommendations patients with pneumothorax are not hypoxaemic and do not F5-F6 require oxygen therapy.

Use a reservoir mask at $15 \mathrm{~L} / \mathrm{min}$ if admitted for observation. Aim at $100 \%$ saturation (oxygen accelerates clearance of pneumothorax if drainage is not required).

Pleural effusions Most patients with pleural effusions are not hypoxaemic. If hypoxaemic, treat by draining the effusion as well as giving oxygen therapy.

Pulmonary embolism Most patients with minor pulmonary embolism are not hypoxaemic and do not require oxygen therapy.

Acute heart failure Consider CPAP or NIV in cases of pulmonary oedema.

Recommendation F4

F

Severe anaemia

The main issue is to correct the anaemia. Most anaemic patients do not require oxygen therapy.

Recommendation F7 Management depends on underlying cause.

Recommendation F8

Recommendations F9-F10

Recommendations F11-12

Postoperative breathlessness

Recommendation J1

COPD, chronic obstructive pulmonary disease; CPAP, continuous positive airway pressure; IMV invasive mechanical ventilation; NIV, non-invasive ventilation; $\mathrm{PCO}_{2}$, arterial or arterialised carbon dioxide tension; $\mathrm{SpO}_{2}$, arterial oxygen saturation measured by pulse oximetry.

outside of healthcare settings, but domiciliary oxygen use by patients is covered by the BTS Guideline for home oxygen use in adults. ${ }^{1-3}$

\section{The scope of the guideline has been widened}

The present guideline includes the following new topics and settings which have been requested by guideline users:

- Postoperative and perioperative care including patient-controlled analgesia.

- Endoscopy and other procedures requiring conscious sedation.

- Palliative care settings including hospices.

- Use of helium-oxygen mixtures (Heliox) and nitrous oxide/oxygen mixtures (Entonox).

- Use of continuous positive airway pressure (CPAP).

- Use of oxygen by healthcare professionals in patients' homes.
- Use of oxygen by voluntary rescue organisations and other non-National Health Service first responders.

- High flow nasal cannulae (HFNC).

\section{The structure and format of the guideline has been} changed since 2008

The 2008 Guideline was published as a self-contained document in Thorax. $^{1}$ Additional educational materials and other resources including audit tools were made available on the British Thoracic Society website. The new guideline exists in two complementary formats.

- A concise guideline which contains recommendations and good practice points is published here.

- The full guideline including evidence review, physiology overview, illustrations and references is published in Thorax ${ }^{1}$ and is available on the British Thoracic Society website. 
Table 3 Conditions for which patients should be monitored closely but oxygen therapy is not required unless the patient is hypoxaemic (section 8.13)

If hypoxaemic, the initial oxygen therapy is nasal cannulae at 2-6 L/min or simple face mask at 5-10 L/min unless saturation is below $\mathbf{8 5 \%}$ (use reservoir mask) or if at risk from hypercapnia (see below).

The recommended initial target saturation range, unless stated otherwise, is $94-98 \%$.

If oximetry is not available, give oxygen as above until oximetry or blood gas results are available.

If patients have COPD or other risk factors for hypercapnic respiratory failure, aim at a saturation of $88-92 \%$ pending blood gas results but adjust to $94-98 \%$ if the $\mathrm{PCO}_{2}$ is normal (unless there is a history of respiratory failure requiring NIV or IMV) and re-check blood gases after 30-60 min, see table 4.

\begin{tabular}{lll}
\hline & Additional comments & Recommendations \\
\hline $\begin{array}{l}\text { Myocardial infarction and acute coronary } \\
\text { syndromes }\end{array}$ & $\begin{array}{l}\text { Most patients with acute coronary artery syndromes are } \\
\text { not hypoxaemic and the benefits/harms of oxygen therapy } \\
\text { are unknown in such cases. Unnecessary use of high }\end{array}$ & Recommendation F13 \\
concentration oxygen may increase infarct size. & \\
Stroke & $\begin{array}{l}\text { Most patients who had stroke are not hypoxaemic. Oxygen } \\
\text { therapy may be harmful for non-hypoxaemic patients with }\end{array}$ \\
mild-moderate strokes. & \\
Hyperventilation or dysfunctional & Exclude organic illness. Patients with pure hyperventilation See section 8.13.3 \\
breathing & due to anxiety or panic attacks are unlikely to require \\
& oxygen therapy. \\
& Re-breathing from a paper bag may cause hypoxaemia \\
& and is not recommended.
\end{tabular}

Most poisonings and drug overdoses (see table 1 for carbon monoxide poisoning)

Hypoxaemia is more likely with respiratory depressant

Recommendation F15

drugs, give antidote if available, for example, naloxone for opiate poisoning.

Check blood gases to exclude hypercapnia if a respiratory depressant drug has been taken. Avoid high blood oxygen levels in cases of acid aspiration as there is theoretical evidence that oxygen may be harmful in this condition. Monitor all potentially serious cases of poisoning in a level 2 or level 3 environment (high-dependency unit or intensive care unit)

Poisoning with paraquat or bleomycin Patients with paraquat poisoning or bleomycin lung injury may be harmed by supplemental oxygen.

Avoid oxygen unless the patient is hypoxaemic.

Target saturation is $85-88 \%$.

Metabolic and renal disorders

Most do not need oxygen

(Tachypnoea may be due to acidosis in these patients)

Acute and subacute neurological and muscular conditions producing muscle These patients may require ventilatory support and they need careful monitoring which includes spirometry. If the patient's oxygen level falls below the target saturation, they need urgent blood gas measurements and are likely to need ventilatory support.

Pregnancy and obstetric emergencies Oxygen therapy may be harmful to the fetus if the mother is not hypoxaemic.

Recommendation F16

Recommendation F17

Recommendation G4 weakness

COPD, chronic obstructive pulmonary disease; IMV, invasive mechanical ventilation; NIV, non-invasive ventilation; $\mathrm{PCO}_{2}$, arterial or arteriolised carbon dioxide tension, $\mathrm{SpO}_{2}$, arterial oxygen saturation measured by pulse oximetry.

\section{INTRODUCTION}

\section{Aim of the guideline}

The key aim of this guideline is to make oxygen use in emergency and healthcare settings safer, simpler and more effective. Oxygen is probably the commonest drug used in the care of patients who present with medical emergencies. Prior to the publication of the first British Thoracic Society Guideline for Emergency Oxygen Use in Adult Patients in 2008, ${ }^{1}$ ambulance teams and emergency department teams were likely to give oxygen to virtually all breathless or seriously ill patients and also to a large number of non-hypoxaemic patients with conditions such as ischaemic heart disease or stroke based on custom and practice. About $34 \%$ of UK ambulance journeys in 2007 involved oxygen use. ${ }^{4}$ This translated to about two million instances of emergency oxygen use per annum by all UK ambulance services, with further use in patients' homes, GP surgeries and in hospitals. Audits of oxygen use and oxygen prescription have shown consistently poor performance in many countries, and most clinicians who deal with medical emergencies have encountered adverse incidents and occasional deaths due to underuse and overuse of oxygen. $^{5-11}$ 
Table 4 COPD and other conditions requiring controlled or low-dose oxygen therapy (section 8.12)

Prior to availability of blood gases, use a $24 \%$ Venturi mask at $2-3 \mathrm{~L} / \mathrm{min}$ or $28 \%$ Venturi mask at $4 \mathrm{~L} / \mathrm{min}$ or nasal cannulae at 1-2 L/min and aim for an oxygen saturation of 88-92\% for patients with risk factors for hypercapnia but no prior history of respiratory acidosis. Adjust target range to $94-98 \%$ if the $\mathrm{PCO}_{2}$ is normal (unless there is a history of previous NIV or IMV) and recheck blood gases after 30-60 min.

\begin{tabular}{|c|c|c|}
\hline & Additional comments & Recommendations \\
\hline $\begin{array}{l}\text { COPD and other conditions causing } \\
\text { fixed airflow obstruction } \\
\text { (eg bronchiectasis) }\end{array}$ & $\begin{array}{l}\text { May need lower range if acidotic or if known to be } \\
\text { very sensitive to oxygen therapy. Ideally use 'Alert } \\
\text { cards' to guide therapy based on previous blood } \\
\text { gas results. Increase Venturi mask flow by up to } \\
50 \% \text { if respiratory rate is above } 30 \mathrm{bpm}\end{array}$ & $\begin{array}{l}\text { Recommendations } \mathrm{G} 1-\mathrm{G} 2 \\
\text { and section } 8.12 .1\end{array}$ \\
\hline Exacerbation of cystic fibrosis & $\begin{array}{l}\text { Admit to regional CF centre if possible, if not } \\
\text { discuss with regional centre or manage according to } \\
\text { protocol agreed with regional CF centre. Ideally use } \\
\text { 'alert cards' to guide therapy. Increase Venturi mask } \\
\text { flow by by up to } 50 \% \text { if respiratory rate is above } \\
30 \text { bpm }\end{array}$ & $\begin{array}{l}\text { Recommendations G1, G3 } \\
\text { and G6 }\end{array}$ \\
\hline $\begin{array}{l}\text { Neuromuscular disease, neurological } \\
\text { condition and chest wall deformity } \\
\text { Morbid obesity }\end{array}$ & $\begin{array}{l}\text { May require ventilatory support. } \\
\text { Risk of hypercapnic respiratory failure }\end{array}$ & $\begin{array}{l}\text { Recommendations G1, G4 } \\
\text { and G6 } \\
\text { Recommendations G1, G5 } \\
\text { and G6 }\end{array}$ \\
\hline
\end{tabular}

$\mathrm{CF}$, Cystic fibrosis; COPD, chronic obstructive pulmonary disease; CPAP, continuous positive airway pressure; IMV, invasive mechanical ventilation; NIV, non-invasive ventilation; $\mathrm{PCO}_{2}$, arterial or arteriolised carbon dioxide tension; $\mathrm{SpO}_{2}$, arterial oxygen saturation measured by pulse oximetry.

\section{Table 5 Abbreviations for oxygen devices for use on bedside charts}

\begin{tabular}{|c|c|c|}
\hline \multicolumn{3}{|c|}{$\begin{array}{l}\text { Add flow in } \mathrm{L} / \mathrm{min} \text { for example, } \mathrm{N} 2 \mathrm{~L} / \mathrm{min}=\text { nasal oxygen at } 2 \mathrm{l} / \mathrm{min} \text {. } \\
\text { V28 } 4 \mathrm{l} / \mathrm{min}=28 \% \text { Venturi mask at } 4 \mathrm{l} / \mathrm{min} \text {. } \\
\text { RM } 15 \mathrm{l} / \mathrm{min}=\text { reservoir mask at } 15 \mathrm{l} / \mathrm{min} \text {. } \\
\text { A, air }\end{array}$} \\
\hline $\mathrm{N}$, nasal cannulae & HFN, high flow nasal cannulae & \\
\hline V24, Venturi mask $24 \%$ & V28, Venturi mask $28 \%$ & V35, Venturi mask 35\% \\
\hline V40, Venturi mask $40 \%$ & V60, Venturi mask $60 \%$ & \\
\hline $\mathrm{H} 28$, humidified $\mathrm{O}_{2} 28 \%$ & $\mathrm{H} 40$, humidified $\mathrm{O}_{2} 40 \%$ & $\mathrm{H} 60$, humidified $\mathrm{O}_{2} 60 \%$ \\
\hline RM, reservoir mask & SM, simple face mask & TM, tracheostomy mask \\
\hline CPAP, continuous positive airway pressure & NIV, non-invasive ventilation & \\
\hline
\end{tabular}

Historically, oxygen has been administered for three main indications of which only one is evidence-based. First, oxygen is given to correct hypoxaemia because severe hypoxaemia is clearly harmful to the human body. Second, oxygen has been administered to ill patients in case they might become hypoxaemic. Recent evidence suggests that, if impaired gas exchange does actually develop, this practice may actually place patients at increased risk (see full Guideline section 6.3). Third, a very high proportion of medical oxygen was administered because most clinicians believed, prior to 2008, that oxygen can alleviate breathlessness in most circumstances. However, there is no good evidence that oxygen relieves breathlessness in non-hypoxaemic patients. There is evidence of lack of effectiveness or minimal effectiveness in mildly hypoxaemic breathless patients with chronic obstructive pulmonary disease (COPD) and advanced cancer (see full Guideline sections 6 and 8.11.4).
Against this background, the Standards of Care Committee of the British Thoracic Society (BTS) established a working party in association with 21 other societies to produce an evidence-based guideline for emergency oxygen use in the UK. This led to the production of the 2008 BTS Guideline for emergency oxygen use in adult patients which was the world's first guideline for emergency oxygen therapy. ${ }^{1}$ This guideline has been implemented throughout the UK and in many other countries leading to over 500 citations in the medical literature up to the end of 2016.

The purpose of the update to the 2008 guideline is to strengthen the evidence base of the previous guideline based on revised methodology (which meets criteria contained in the AGREE II Instrument) and to extend the evidence base to the end of $2013 .{ }^{12}$ Additionally, the remit of the 2008 Guideline has been broadened to cover several new aspects of oxygen use and a broader range of locations where oxygen might be used. 
See patient's drug chart and Chart 1 and tables 1-4 for starting dose and target saturation Choose the most suitable delivery system and flow rate

Titrate oxygen up or down to maintain the target oxygen saturation.

The table below shows available options for stepping dosage up or down.

The chart does NOT imply any equivalence of dose between Venturi masks and nasal cannulae.

Allow at least 5 minutes at each dose before adjusting further upwards or downwards

(except with major and sudden fall in saturation - falls $\geq 3 \%$ also require clinical review)

Once your patient has adequate and stable saturation on minimal oxygen dose, consider discontinuation of oxygen therapy.

Seek medical advice if
patient appears to
need increasing
oxygen therapy or if
there is a rising NEWS
or Track and Trigger
score.

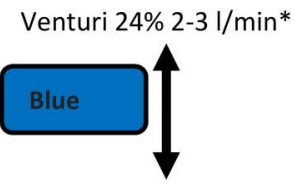

Venturi $28 \% 4-6 \mathrm{I} / \mathrm{min}$

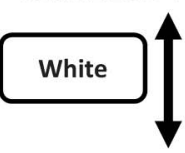

Venturi 35\% 8-12 l/min

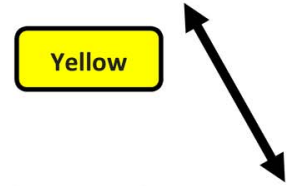

$$
\begin{aligned}
& \text { All Patients must } \\
& \text { have ABG or Earlobe } \\
& \text { Blood Gases (ELBG) } \\
& \text { within } 1 \text { hour of } \\
& \text { requiring increased } \\
& \text { oxygen dose. }
\end{aligned}
$$

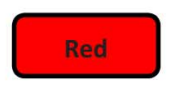
Venturi $40 \% 10-15$
$\mathrm{l} / \mathrm{min}$

Nasal cannulae or

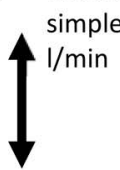

Green
Nasal cannulae $1 \mathrm{l} / \mathrm{min}$

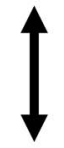

Nasal cannulae $2 \mathrm{l} / \mathrm{min}$

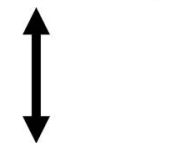

Nasal cannulae $4 \mathrm{l} / \mathrm{min}$

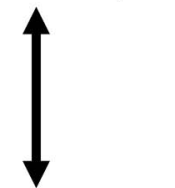

imple face mask at 5-6

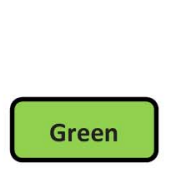

Venturi 60\% 12-15

or Simple face mask

7-10 l/min

Signs of Respiratory Deterioration

- $\quad \hat{~ R e s p ~ R a t e ~}$ (especially if $>30$ )

- $\sqrt{\mathrm{SpO} 2}$

- 仓ि oxygen dose needed to keep $\mathrm{SpO} 2$ in target range

- 仓ि EWS/Trigger score

$\mathrm{CO}_{2}$ Retention

- Drowsiness

- Headache

- Flushed face

- Flapping Tremor

Seek Medical Advice

Reservoir mask at $15 \mathrm{l} /$ min Oxygen

flow

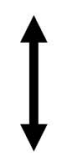

If Reservoir mask required, seek senior medical input immediately

* For Venturi masks, the higher flow rate is required if the respiratory rate is $\mathbf{3 0}$

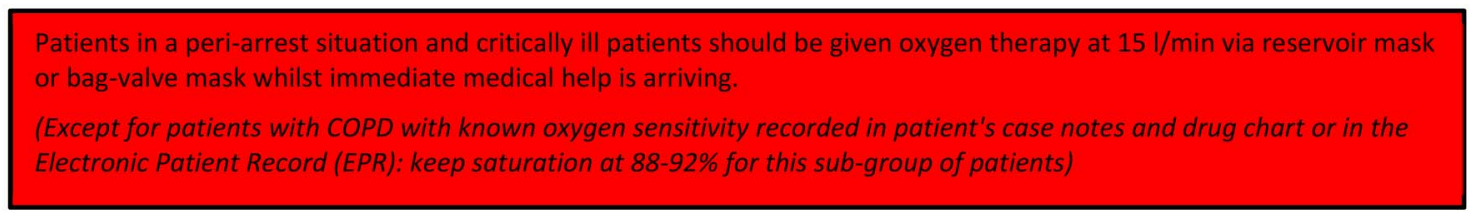

Figure 2 Flow chart for oxygen administration on general wards in hospitals. COPD, chronic obstructive pulmonary disease. EPR, electronic patient record; EWS, early warning score; NEWS, National Early Warning Score; SpO2, arterial oxygen saturation measured by pulse oximetry. 


\section{Table 6 SIGN evidence levels}

\section{SIGN levels of evidence}

$1++$ High-quality meta-analyses, systematic reviews of RCTs or RCTs with a very low risk of bias

1+ Well-conducted meta-analyses, systematic reviews or RCTs with a low risk of bias

1 Meta-analyses, systematic reviews or RCTs with a high risk of bias

2++ High-quality systematic reviews of case-control or cohort studies

High-quality case-control or cohort studies with a very low risk of confounding or bias and a high probability that the relationship is causal

2+ Well-conducted case-control or cohort studies with a low risk of confounding or bias and a moderate probability that the relationship is causal

2- Case-control or cohort studies with a high risk of confounding or bias and a significant risk that the relationship is not causal

3 Non-analytic studies, eg, case reports, case series

4 Expert opinion

$\mathrm{RCT}$, randomised controlled trial.

\section{Table 7 SIGN grades of recommendation}

\section{Grades of recommendations}

A At least one meta-analysis, systematic review or RCT rated as 1++, and directly applicable to the target population; or A body of evidence consisting principally of studies rated as $1+$, directly applicable to the target population, and demonstrating overall consistency of results

B A body of evidence including studies rated as $2++$, directly applicable to the target population, and demonstrating overall consistency of results; or Extrapolated evidence from studies rated as $1++$ or $1+$

C A body of evidence including studies rated as $2+$, directly applicable to the target population and demonstrating overall consistency of results; or Extrapolated evidence from studies rated as 2++

D Evidence level 3 or 4 ; or Extrapolated evidence from studies rated as $2+$

Good practice points

$\checkmark$ Recommended best practice based on the clinical experience of the guideline development group

\section{Intended users of the guideline and target patient populations}

This guideline is mainly intended for use by all healthcare professionals who may be involved in emergency oxygen use. This will include ambulance staff, first responders, paramedics, doctors, nurses, midwives, physiotherapists, pharmacists and all other healthcare professionals who may deal with ill or breathless patients. Advice is also provided for first responders belonging to voluntary organisations or other non-NHS bodies. Information based on this guideline is available on the BTS website for use in the following situations:

- Hospital use.

- Primary care use.

- Ambulance use (supplemented by Ambulance service guidance based on this guideline) ${ }^{13}$

- Use by nursing staff and allied health professions.

These abbreviated versions of the guideline contain the key recommendations and tables and charts that are relevant to the particular situation.

\section{Areas covered by this guideline}

The guideline addresses the use of oxygen in three main categories of adult patients in the prehospital and hospital setting and in other settings such as palliative care:

- Critically ill or hypoxaemic patients.

- Hypoxaemic patients and patients at risk of hypoxaemia.

- Non-hypoxaemic patients who may benefit from oxygen (eg, carbon monoxide poisoning).

\section{Areas not covered by this guideline}

- Oxygen use in paediatrics: the present guideline applies only to patients aged $>16$ years.

- Oxygen use for high altitude activities.

- Oxygen use during air travel.

- Underwater diving and diving accidents.

- Oxygen use in animal experiments.

- Oxygen use in high-dependency units.

- Oxygen use in intensive care units.

- Interhospital level 3 transfers.

- Hyperbaric oxygen.

- Respiratory support techniques including tracheal intubation, invasive ventilation and non-invasive ventilation (NIV) (CPAP is included).

- Self-initiated use of oxygen by patients who have home oxygen for any reason.

- Ongoing care of hypoxaemic patients at home. 


\section{Limitations of the guideline}

This guideline is based on the best available evidence concerning oxygen therapy. However, a guideline can never be a substitute for clinical judgement in individual cases. There may be cases where it is appropriate for clinicians to act outwith the advice contained in this guideline because of the needs of individual patients, especially those with complex or interacting disease states. Furthermore, the responsibility for the care of individual patients rests with the clinician in charge of the patient's care and the advice offered in this guideline must, of necessity, be of a general nature and should not be relied on as the only source of advice in the treatment of individual patients. In particular, this guideline gives very little advice about the management of the many medical conditions that may cause hypoxaemia (apart from the specific issue of managing the patients' hypoxaemia). Readers are referred to other guidelines for advice on the management of specific conditions such as COPD, pneumonia, heart failure etc. Some of these disease-specific guidelines may suggest slightly different approaches to emergency oxygen therapy whereas the present guideline aims to provide simple all-embracing advice about oxygen therapy.

\section{Definitions of terms used in the guideline (and normal values)}

Terms such as hypoxaemia and normal values for oxygen and carbon dioxide in blood gases are provided in the full Guideline (sections 3-6).

\section{Planned review and updating of the guideline}

The 2017 guideline will be reviewed by BTS within 5 years from publication.

\section{Declarations of interest}

All members of the Guideline Group made declarations of interest in line with the BTS Policy, and further details can be obtained on request from BTS.

\section{SUMMARY OF GUIDELINE RECOMMENDATIONS AND GOOD PRACTICE POINTS}

A. Achieving desirable oxygen saturation ranges in acute illness (see figures 1-2 and full Guideline sections 6 and 8)

A1. This guideline recommends aiming to achieve a normal or near-normal oxygen saturation for all acutely ill patients apart from those at risk of hypercapnic respiratory failure (grade $\mathrm{D}$ ).

A2. The recommended target saturation range for acutely ill patients not at risk of hypercapnic respiratory failure is $94-98 \%$ (grade $\mathrm{D}$ ).

A3. For most patients with known COPD or other known risk factors for hypercapnic respiratory failure (eg, morbid obesity, cystic fibrosis, chest wall deformities or neuromuscular disorders, or fixed airflow obstruction associated with bronchiectasis), a target saturation range of $88-92 \%$ is suggested pending the availability of blood gas results (grade A for COPD, grade $\mathrm{D}$ for other conditions).

A4. Most non-hypoxaemic breathless patients do not benefit from oxygen therapy, but a sudden reduction of $\geq 3 \%$ in a patient's oxygen saturation within the target saturation range should prompt fuller assessment of the patient (and the oximeter signal) because this may be the first evidence of an acute illness (grade D).

A5 Since oxygenation is reduced in the supine position, fully conscious hypoxaemic patients should ideally be allowed to maintain the most upright posture possible (or the most comfortable posture for the patient) unless there are good reasons to immobilise the patient (eg, skeletal or spinal trauma) (grade D).

B. Clinical and laboratory assessment of hypoxaemia and hypercapnia (see full Guideline section 7)

B1 Fully trained clinicians should assess all acutely ill patients by measuring respiratory rate, pulse rate, blood pressure, temperature and assessing circulating blood volume and anaemia. Expert assistance from specialists in intensive care or from other disciplines should be sought at an early stage if patients are thought to have major lifethreatening illnesses and clinicians should be prepared to call for assistance when necessary including a call for a 999 ambulance in prehospital care or a call for the resuscitation team or intensive care unit (ICU) outreach team in hospital care (grade D).

B2. Oxygen saturation, 'the fifth vital sign', should be checked by trained staff using pulse oximetry in all breathless and acutely ill patients (supplemented by blood gases when necessary) and the inspired oxygen device and flow rate should be recorded on the observation chart with the oximetry result (grade $\mathrm{D}$ ).

B3. Initial clinical assessment and subsequent monitoring of acutely unwell patients should include the use of a recognised physiological 'track and trigger' system, such as the NEWS which may trigger clinical review due to hypoxaemia, need for supplementary oxygen or for other reasons (grade D).

B4. For patients who are at risk of hypercapnic respiratory failure, it is recommended that the relevant section of the 2017 NEWS chart should be used. Points are awarded if the oxygen saturation is below or above the target range (grade $\mathrm{D}$ ).

Good practice points for clinical assessment of patients with suspected hypoxaemia

$\checkmark$ The medical history should be taken when possible in an acutely breathless patient and may point to the diagnosis of a particular acute illness such as pneumonia or pulmonary embolism or an exacerbation of a chronic condition such as COPD, asthma or heart failure.

$\checkmark$ Never discontinue oxygen therapy to obtain an oximetry measurement on room air in patients who clearly require oxygen therapy. 
$\checkmark$ Physical examination should be undertaken urgently. This may provide evidence of a specific diagnosis such as heart failure or a large pleural effusion, but it is common for the cause of breathlessness to remain undiagnosed until the results of tests such as chest radiographs are available.

$\checkmark$ Record arterial oxygen saturation measured by pulse oximetry $\left(\mathrm{SpO}_{2}\right)$ and consider blood gas assessment in patients with unexplained confusion and agitation as this may be presenting feature of hypoaxaemia and/or hypercapnia (cyanosis is a difficult physical sign to record confidently, especially in poor light or with an anaemic or plethoric patient).

$\checkmark$ Carefully measure respiratory rate and heart rate because tachypnoea and tachycardia are more common than a physical finding of cyanosis in hypoxaemic patients.

$\checkmark$ Appropriate changes should be made to any 'track and trigger' system used to allow for a lower target range in patients at risk of hypercapnic respiratory failure. These patients should score no points for saturation if within their target range and they should score points if the oxygen saturation falls below the target range or if the saturation rises above the target range while breathing oxygen. The 2017 update of the national NEWS chart has a special section for oximetry measurements for use with patients who have a target range $88-92 \%$ and it is recommended that the 2017 NEWS chart should be used in all hospitals (see recommendation $\mathrm{B} 4$ ).

$\checkmark$ The presence of a normal $\mathrm{SpO}_{2}$ does not negate the need for blood gas measurements especially if the patient is on supplemental oxygen therapy. Pulse oximetry will be normal in a patient with normal oxygen tension $\left(\mathrm{PO}_{2}\right)$ but abnormal blood $\mathrm{pH}$ or carbon dioxide tension $\mathrm{PCO}_{2}$ or with a low blood oxygen content due to anaemia. For this reason, blood gases and full blood count tests are required as early as possible in all situations where these measurements may affect patient outcomes.

$\checkmark$ All clinical staff who use oximeters must be trained in their use and made aware of the limitations of oximetry. (Oximetry is a valuable clinical tool but subject to artefact and errors of interpretation).

C. Arterial and capillary blood gases (see full Guideline sections 7.1.3 and 8.4 and 8.5)

C1. For critically ill patients or those with shock or hypotension (systolic blood pressure $<90 \mathrm{~mm} \mathrm{Hg}$ ), the initial blood gas measurement should be obtained from an arterial sample. For most patients who require blood gas sampling, either arterial blood gases or arterialised earlobe blood gases may be used to obtain an accurate measure of $\mathrm{pH}$ and $\mathrm{PCO}_{2}$. However, the $\mathrm{PO}_{2}$ is less accurate in earlobe blood gas samples (it underestimates the oxygen tension by $0.5-1 \mathrm{kPa}$ ) so oximetry should be monitored carefully if earlobe blood gas specimens are used and a repeat arterial specimen should be taken if there is any concern about the accuracy of a capillary sample (grade D).

C2. Local anaesthesia should be used for all arterial blood gas specimens except in emergencies (grade A).

C3. Blood gases should be checked in the following situations:

- All critically ill patients (Grade D).

- Unexpected or inappropriate fall in $\mathrm{SpO}_{2}$ below $94 \%$ in patients breathing air or oxygen or any patient requiring oxygen to achieve the above target range. (Allowance should be made for transient dips in saturation to $90 \%$ or less in normal individuals during sleep) (grade D).

- Deteriorating oxygen saturation (fall of $\geq 3 \%$ ) or increasing breathlessness in a patient with previously stable chronic hypoxaemia (eg, severe COPD) (grade D).

- Most previously stable patients who deteriorate clinically and require increased fraction of inspired oxygen $\left(\mathrm{FiO}_{2}\right)$ to maintain a constant oxygen saturation (grade $\mathrm{D}$ ).

- Any patient with risk factors for hypercapnic respiratory failure who develops acute breathlessness, deteriorating oxygen saturation, drowsiness or other symptoms of carbon dioxide retention (grade D).

- Patients with breathlessness who are thought to be at risk of metabolic conditions such as diabetic ketoacidosis or metabolic acidosis due to renal failure (grade D).

- Any other evidence from the patient's medical condition that would indicate that blood gas results would be useful in the patient's management (eg, an unexpected change in 'track and trigger' systems such as a sudden rise of several units in the NEWS score or an unexpected fall in oxygen saturation of $3 \%$ or more, even if within the target range) (grade $\mathrm{D}$ ).

Good practice point: patients requiring increased concentration of oxygen

$\checkmark$ The requirement for an increased concentration of oxygen is an indication for urgent clinical reassessment of the patient (and repeat blood gas measurements in most instances, see recommendations W13 and W18 for exceptions).

D. Initial oxygen therapy; initial choice of equipment for patients who do not have critical illness (see figures 1-2 and table 2 and full Guideline sections 8.9 and 10) ${ }^{\mathrm{i}}$

Initial oxygen therapy in critical illness is covered in the next section.

D1. For acutely breathless patients not at risk of hypercapnic respiratory failure who have saturations below $85 \%$, treatment should be started with a reservoir mask at $15 \mathrm{~L} / \mathrm{min}$ in the first instance. The oxygen concentration can be adjusted downwards (using nasal cannulae at $1-6 \mathrm{~L} / \mathrm{min}$ or a simple face mask at $5-10 \mathrm{~L} / \mathrm{min}$ ) to maintain a target saturation of 94-98\% once the patient has stabilised (grade D).

${ }^{\mathrm{i}}$ For initial management of patients at risk of hypercapnic respiratory failure, see recommendations G1 and G2. 
D2. In other cases of acute hypoxaemia without critical illness or risk factors for hypercapnic respiratory failure, treatment should be started with nasal cannulae (or a simple face mask if cannulae are not tolerated or not effective) with the flow rate adjusted to achieve a saturation of 94-98\% (grade D).

D3. If medium-concentration therapy with nasal cannulae or a simple face mask does not achieve the desired saturation, change to a reservoir mask and seek senior or specialist advice (grade D).

Good practice point

$\checkmark$ High flow nasal oxygen using specialised equipment should be considered as an alternative to reservoir mask treatment in patients with acute respiratory failure without hypercapnia. For initial management of patients at risk of hypercapnic respiratory failure see recommendations G1 and G2.

E. Oxygen therapy in critical illness (see table 1 and full Guideline section 8.10)

E1. Use the highest feasible inspired oxygen for ventilation during cardiopulmonary resuscitation. Once spontaneous circulation has returned and arterial blood oxygen saturation can be monitored reliably, aim for a target saturation range of $94-98 \%$ and take an arterial blood gas sample to guide on-going oxygen therapy. If the blood gas shows hypercapnic respiratory failure, reset the target range to $88-92 \%$ or consider mechanical ventilation (grade D).

E2. In critical illness, including major trauma, sepsis, shock and anaphylaxis, initiate treatment with a reservoir mask at $15 \mathrm{~L} / \mathrm{min}$ and aim at a saturation range of 94-98\%. This advice also applies to patients with critical illness who have risk factors for hypercapnia pending the results of blood gas measurements and expert assessment. In patients with spontaneous circulation and a reliable oximetry reading, it may be possible to maintain a saturation of $94-98 \%$ using lower concentrations of oxygen (grade D).

E3. In cases of drowning, aim at an oxygen saturation of 94-98\% once spontaneous circulation is restored (grade D).

E4. In patients with acute seizures due to epilepsy or other causes, high-concentration oxygen should be administered until a satisfactory oximetry measurement can be obtained and clinicians should then aim for an oxygen saturation of $94-98 \%$ or $88-92 \%$ if the patient is at risk of hypercapnic respiratory failure (grade D).

E5. In cases of major head injury, aim at an oxygen saturation of 94-98\%. Initial treatment should involve high-concentration oxygen from a reservoir mask at $15 \mathrm{~L} / \mathrm{min}$ pending availability of satisfactory blood gas measurements or until the airway is secured by intubation (grade D).

E6. In cases of carbon monoxide poisoning, an apparently 'normal' oximetry reading may be produced by carboxyhaemoglobin, so aim at an oxygen saturation of $100 \%$ and use a reservoir mask at $15 \mathrm{~L} / \mathrm{min}$ irrespective of the oximeter reading and $\mathrm{PaO}_{2}$ (grade D).

F. Oxygen therapy for specific conditions that frequently require oxygen therapy (see tables 2 and 3 and full Guideline sections 8.11 and 8.13 )

Respiratory conditions with low risk of hypercapnic respiratory failure:

F1. In acute asthma, aim at an oxygen saturation of 9498\% (grade D).

F2. In cases of pneumonia who are not at risk of hypercapnic respiratory failure, aim at an oxygen saturation of $94-98 \%$ (grade D).

F3. In acute breathlessness due to lung cancer, aim at an oxygen saturation of $94-98 \%$ unless there is coexisting COPD. See also 'Oxygen use in palliative care' section (grade $\mathrm{D}$ ).

F4. In acute deterioration of pulmonary fibrosis or other interstitial lung diseases, aim at an oxygen saturation of 94-98\% or the highest possible if these targets cannot be achieved (grade D).

F5. In most cases of pneumothorax, aim at an oxygen saturation of $94-98 \%$ if the patient is not at risk of hypercapnic respiratory failure (grade $\mathrm{D}$ ).

F6. In patients with pneumothorax having hospital observation without drainage, the use of high concentration oxygen $(15 \mathrm{~L} / \mathrm{min}$ flow rate via reservoir mask) is recommended unless the patient is at risk of hypercapnic respiratory failure (grade D).

F7. In pleural effusion, aim at an oxygen saturation of 94-98\% (or $88-92 \%$ if the patient is at risk of hypercapnic respiratory failure) (grade D).

F8. In pulmonary embolism, aim at an oxygen saturation of $94-98 \%$ (or $88-92 \%$ if the patient is at risk of hypercapnic respiratory failure) (grade D).

Non-respiratory conditions:

F9. In acute heart failure, aim at an oxygen saturation of 94-98\% (or $88-92 \%$ if the patient is at risk of hypercapnic respiratory failure) (grade D).

F10. Continuous positive airway pressure (CPAP) with entrained oxygen or high-flow humidified nasal oxygen to maintain saturation $94-98 \%$ (or $88-92 \%$ if at risk of hypercapnia) should be considered as an adjunctive treatment to improve gas exchange in patients with cardiogenic pulmonary oedema who are not responding to standard treatment (or noninvasive ventilation (NIV) if there is co-existant hypercapnia and acidosis) (grade B).

F11. In anaemia, aim at an oxygen saturation of $94-98 \%$ or $88-92 \%$ if the patient is at risk of hypercapnic respiratory failure (grade $\mathrm{D}$ ).

Good practice point

$\checkmark$ Correction of anaemia by blood transfusion should be based on national guidelines.

F12. In sickle cell crisis and acute chest syndrome, aim for an oxygen saturation of $94-98 \%$ or aim at the saturation level that is usual for the individual patient (grade D). 
Good practice point regarding sickle cell crisis

$\checkmark$ Arterial or arterialised capillary blood gases should be sampled if there is any doubt about the reliability of oximetry during a sickle cell crisis.

F13. In myocardial infarction and acute coronary syndromes, aim at an oxygen saturation of 94-98\% or $88-92 \%$ if the patient is at risk of hypercapnic respiratory failure (grade $\mathrm{D}$ ).

F14. High concentrations of oxygen should be avoided in patients with stroke, unless required to maintain normal oxygen saturation. Aim at an oxygen saturation of $94-98 \%$ or $88-92 \%$ if the patient is at risk of hypercapnic respiratory failure (grade D).

Good practice points regarding stroke management

$\checkmark$ Oxygen saturation should be monitored at least every 4 hours throughout the day and night in patients with acute stroke and all episodes of hypoxaemia treated.

$\checkmark$ Patients with hypoxaemia post-stroke require medical review to establish and treat the cause.

$\checkmark$ Oxygen should only be given once the airway has been cleared and at the lowest concentration necessary to achieve an oxygen saturation of $94-98 \%$ or $88-92 \%$ if the patient is at risk of hypercapnic respiratory failure.

$\checkmark$ Oxygen should be given via nasal cannulae, unless there are clear indications for a different oxygen delivery system.

$\checkmark$ Patients with stroke and cardiorespiratory comorbidities should be positioned as upright as possible, in a chair if possible (see recommendation A5).

$\checkmark$ Patients with a reduced level of consciousness after stroke should be nursed in the recovery position with the paralysed side lowest.

Suspected hyperventilation:

Good practice points regarding patients with suspected hyperventilation

$\checkmark$ Organic illness must be excluded before making a diagnosis of hyperventilation.

$\checkmark$ Patients with a definite diagnosis of hyperventilation should have their oxygen saturation monitored. Those with normal or high $\mathrm{SpO}_{2}$ do not require oxygen therapy.

$\checkmark$ Rebreathing from a paper bag can be dangerous and is NOT advised as a treatment for hyperventilation.

F15. In most poisonings, aim at an oxygen saturation of 94-98\% unless the patient is at risk of hypercapnic respiratory failure (grade $\mathrm{D}$ ).

F16. In poisoning by paraquat and poisoning by bleomycin, give oxygen only if the saturation falls below $85 \%$ and reduce or stop oxygen therapy if the saturation rises above $88 \%$ (grade $\mathrm{D}$ ).

F17. In most metabolic and renal disorders, aim at an oxygen saturation of $94-98 \%$ unless the patient is at risk of hypercapnic respiratory failure (grade D).

F18. For patients with cluster headaches, oxygen should be administered using a flow of at least $12 \mathrm{~L} / \mathrm{min}$ from a reservoir mask and home oxygen should be provided (grade D).

G. Patients at risk of hypercapnic respiratory failure (see table 4 and full Guideline section 8.12)

G1. (also A3) For most patients with known COPD or other known risk factors for hypercapnic respiratory failure (eg, morbid obesity, cystic fibrosis, chest wall deformities or neuromuscular disorders or fixed airflow obstruction associated with bronchiectasis), a target saturation range of $88-92 \%$ is suggested pending the availability of blood gas results (grade A for COPD, grade D for other conditions).

G2. Some patients with COPD and other conditions are vulnerable to repeated episodes of hypercapnic respiratory failure. In these cases, it is recommended that treatment should be based on the results of previous blood gas estimations during acute exacerbations. For patients with prior hypercapnic failure (requiring NIV or intermittent positive pressure ventilation) who do not have an alert card, it is recommended that low-concentration oxygen treatment should be started using a $24 \%$ Venturi mask at $2-$ $3 \mathrm{~L} / \mathrm{min}$ (or a $28 \%$ Venturi mask at $4 \mathrm{~L} / \mathrm{min}$ or nasal cannulae at $1-2 \mathrm{~L} / \mathrm{min}$ if a $24 \%$ mask is not available) with an initial target saturation of $88-92 \%$ pending urgent blood gas results. These patients should be treated as a high priority by emergency services, and the oxygen concentration should be reduced if the saturation exceeds $92 \%$ but increased if it falls below $88 \%$ (grade D).

Good practice points for COPD and other conditions that may cause hypercapnic respiratory failure

Diagnosis of COPD or suspected exacerbation of COPD:

$\checkmark$ If the diagnosis is unknown, patients over 50 years of age who are long-term smokers with a history of chronic breathlessness on minor exertion such as walking on level ground and no other known cause of breathlessness should be treated as having suspected COPD for the purposes of this guideline.

$\checkmark$ Spirometry should be measured at least once during hospital admissions for suspected COPD (as per the National Institute of Health and Care Excellence (NICE) COPD Guideline).$^{15}$ Measurement of spirometry may confirm (or exclude) a diagnosis of airflow obstruction, and the FEV1 level is a useful indicator of disease severity in COPD.

Immediate management of patients with known or suspected COPD:

$\checkmark$ If the saturation remains below $88 \%$ in prehospital care despite a $28 \%$ Venturi mask, change to nasal cannulae at $2-6 \mathrm{~L} / \mathrm{min}$ or a simple face mask at $5 \mathrm{~L} /$ min with target saturation of $88-92 \%$ and alert the emergency department that the patient is to be treated as a high priority.

$\checkmark$ Patients with a respiratory rate $>30 \mathrm{bpm}$ should have the flow rate from Venturi masks set above the minimum flow rate specified for the Venturi mask packaging to compensate for the patient's increased 
inspiratory flow. Increasing the oxygen flow rate into a Venturi mask does not increase the concentration of oxygen which is delivered.

$\checkmark$ Patients with a significant likelihood of severe COPD or other illness that may cause hypercapnic respiratory failure should be triaged as very urgent on arrival in hospital emergency departments and blood gases should be measured on arrival in hospital.

$\checkmark$ Prior to availability of blood gas measurements, use a $24 \%$ Venturi mask at $2-3 \mathrm{~L} / \mathrm{min}$ or nasal cannulae at $1-2 \mathrm{~L} / \mathrm{min}$ or $28 \%$ Venturi mask at $4 \mathrm{~L} / \mathrm{min}$ and aim for an oxygen saturation of $88-92 \%$

Initial hospital management of patients with exacerbation of COPD:

$\checkmark$ Patients with exacerbations of COPD need careful monitoring for hypercapnic respiratory failure with respiratory acidosis which may develop in the course of a hospital admission even if the initial blood gases were satisfactory.

$\checkmark$ Avoid excessive oxygen use in patients with COPD. The risk of respiratory acidosis in patients with hypercapnic respiratory failure is increased if the $\mathrm{PaO}_{2}$ is above $10.0 \mathrm{kPa}$ due to previous excessive oxygen use.

$\checkmark$ If following blood gas measurements the $\mathrm{pH}$ and $\mathrm{PCO}_{2}$ are normal, aim for an oxygen saturation of 94-98\% unless there is a history of previous hypercapnic respiratory failure requiring NIV or intermittent positive pressure ventilation or if the patient's usual oxygen saturation when clinically stable is below $94 \%$ (these patients should have a target range of $88-92 \%$ ). Blood gases should be repeated at 30-60 min to check for rising $\mathrm{PCO}_{2}$ or falling $\mathrm{pH}$.

$\checkmark$ Recheck blood gases after $30-60$ min (or if there is evidence of clinical deterioration) for all patients with COPD or other risk factors for hypercapnic respiratory failure even if the initial $\mathrm{PCO}_{2}$ measurement was normal.

$\checkmark$ If the $\mathrm{PCO}_{2}$ is raised but $\mathrm{pH}$ is $\geq 7.35 \quad([\mathrm{H}+]$ $\leq 45 \mathrm{nmol} / \mathrm{L}$ ) and/or a high bicarbonate level $(>28 \mathrm{mmol} / \mathrm{L})$, the patient has probably got longstanding hypercapnia; maintain target range of 8892\% for these patients. Blood gases should be repeated at 30-60 min to check for rising $\mathrm{PCO}_{2}$ or falling $\mathrm{pH}$.

$\checkmark$ If the patient is hypercapnic $\left(\mathrm{PCO}_{2}>6 \mathrm{kPa}\right.$ or 45 $\mathrm{mm} \mathrm{Hg}$ ) and acidotic $(\mathrm{pH}<7.35$ or $[\mathrm{H}+]>45 \mathrm{nmol} / \mathrm{L})$, start NIV with targeted oxygen therapy if respiratory acidosis persists for more than 30 min after initial standard medical management.

$\checkmark$ For patients using Venturi masks, consider changing from Venturi mask to nasal cannulae once the patient has stabilised.

$\checkmark$ For patients who use long-term home oxygen (LTOT) for severe COPD, a senior clinician should consider setting a patient-specific target range if the standard range of $88-92 \%$ would require inappropriate adjustment of the patient's usual oxygen therapy while the patient is in hospital.
Good practice point

Management of hypercapnia or respiratory acidosis due to excessive oxygen therapy (avoidance of life-threatening rebound hypoxaemia):

$\checkmark$ If a patient is suspected to have hypercapnic or respiratory failure due to excessive oxygen therapy, the oxygen therapy must be stepped down to the lowest level required to maintain a saturation range of $88-92 \%$. This may be achieved using $28 \%$ or $24 \%$ oxygen from a Venturi mask or 1-2 L/min via nasal cannulae depending on oxygen saturation and subsequent blood gas measurements.

$\checkmark$ Sudden cessation of supplementary oxygen therapy can cause life-threatening rebound hypoxaemia with a rapid fall in oxygen saturations below the starting oxygen saturation prior to the start of supplementary oxygen therapy.

G3. Initial oxygen treatment of cystic fibrosis exacerbations should be similar to the initial oxygen treatment of COPD exacerbations with target saturation 88-92\% (see sections 8.12.1-8.12.2) (grade D).

G4. In the initial management of musculoskeletal and neurological disorders with acute respiratory failure, or acute-on-chronic respiratory failure, aim at an oxygen saturation of $88-92 \%$ and measure blood gases to determine if NIV will be required (grade D).

Good practice point regarding patients with neurological disorders

$\checkmark$ Patients with respiratory failure due to neurological disorders or muscle disease are at high risk of dying and require urgent assessment to determine if they are likely to require non-invasive or invasive ventilator support rather than oxygen therapy. Monitor these patients with blood gases and regular spirometry (forced vital capacity). Patients' wishes regarding this form of treatment should established as early as possible in the course of the illness, ideally before an acute episode has developed.

G5. Morbidly obese patients (body mass index (BMI) $>40 \mathrm{~kg} / \mathrm{m}^{2}$ ), even without evidence of coexistent obstructive sleep apnoea are at risk of hypoventilation and should be given titrated oxygen to maintain a target saturation of 88-92\% (grade D).

G6. NIV should be considered for hypercapnic patients with COPD, CF, neuromuscular disorders or morbid obesity who are at risk of hypercapnic respiratory failure if the $\mathrm{pH}$ is $<7.35$ or $[\mathrm{H}+]>45 \mathrm{nmol} / \mathrm{L}$ (grade D) (see BTS/ICS Guidelines for the Ventilatory Management of Acute Hypercapnic Respiratory Failure in Adults). ${ }^{16}$

H. Oxygen use during pregnancy (see full Guideline section 8.14)

H1. Women who suffer from major trauma, sepsis or acute illness during pregnancy should receive the same oxygen therapy as any other seriously ill patients, with a target oxygen saturation of 94-98\%. The same target range should be applied to women with hypoxaemia due to acute complications of 
pregnancy (eg, collapse related to amniotic fluid embolus, eclampsia or antepartum or postpartum haemorrhage) (grade D).

H2. Women with underlying hypoxaemic conditions (eg, heart failure) should be given supplemental oxygen during labour to achieve an oxygen saturation of 94-98\% unless they are at risk of hypercapnic respiratory failure (target range $88-92 \%$ ) (grade D).

H3. Pregnant women who are fully conscious with no cardiovascular compromise may be managed in the sitting position or if lying down should use the full left lateral position (grade D).

H4. Pregnant women above 20 weeks gestation (uterine fundus at or above the level of the umbilicus) who are at risk of developing associated cardiovascular compromise (eg, trauma and vaginal bleeding) should be positioned to avoid aortocaval compression by using left lateral tilt, manual uterine displacement or by placing them in a full left lateral position (grade D).

H5. Women who are more than 20 weeks pregnant with evidence of hypoxaemia associated with reduced consciousness or those requiring respiratory or cardiovascular support or cardiopulmonary resuscitation should be managed with left lateral tilt or manual uterine displacement (ideally to the left) to improve cardiac output and oxygen delivery (grade D).

H6. The use of oxygen supplementation during intrauterine fetal resuscitation during labour was widespread in the past, but there is no evidence of benefit. There is weak evidence of harm to the fetus if supplemental oxygen is given for long periods during uncomplicated labour. Overall, the use of oxygen during labour is only required when there is evidence of maternal hypoxaemia (oxygen saturation $<94 \%$ ) (grade D).

J. Oxygen use in perioperative care and during procedures requiring conscious sedation (see full Guideline sections 8.158.16 and 10.11)

J1. Hyperoxaemia is not recommended routinely in the perioperative and postoperative period to reduce the incidence of postoperative nausea and vomiting (grade D).

J2. All procedures involving conscious sedation warrant routine continuous monitoring of oxygen saturation via pulse oximetry prior and during the procedure, and in the recovery period, particularly fibre-optic bronchoscopy and upper GI endoscopy where a reduction in arterial oxygen saturation $\left(\mathrm{SaO}_{2}\right)$ is common, particularly with concurrent use of sedation (grade $\mathrm{C}$ ).

J3. Significant arterial oxygen desaturation $\left(\mathrm{SpO}_{2}<90 \%\right.$ or fall of $4 \%$ or more that is prolonged $(>1 \mathrm{~min}$ during endoscopy procedures) should be corrected by supplemental oxygen with the aim of achieving target oxygen saturations of $94-98 \%$, or $88-92 \%$ in those at risk of hypercapnic respiratory failure (grade D).
J4. Complicated upper GI endoscopy or procedures in patients with cardiorespiratory comorbidity are especially likely to lead to hypoxaemia and may also lead to hypercapnia, especially if the patient is heavily sedated. It is recommended that blood gases should be measured if such patients should require prolonged oxygen administration. The routine administration of oxygen is not recommended as it may delay the recognition of respiratory failure (grade $\mathrm{D}$ ).

J5. Constant clinical assessment of the patient is crucial at all stages of conscious sedation procedures and monitoring of capnography or transcutaneous carbon dioxide levels may be a useful adjunct to identify early respiratory depression (grade D).

J6. During the recovery period after procedures requiring conscious sedation, supplemental oxygen should be titrated to achieve target saturations of $94-98 \%$ in most patients and $88-92 \%$ in those at risk of hypercapnic respiratory failure (see 10.5.1) (grade D).

Good practice points related to oxygen use in perioperative care

$\checkmark$ A target saturation of $94-98 \%$ is recommended for most surgical patients except those at risk of hypercapnic respiratory failure when a range of $88-92 \%$ should be achieved.

$\checkmark$ Pulse oximetry monitoring is recommended for postoperative patients despite the lack of evidence from randomised studies.

$\checkmark$ Patients using patient-controlled analgesia (PCA) should have two-hourly oximetry observations because of the risk of hypoxaemia. Oxygen should be administered to keep patients within the appropriate target saturation range.

$\checkmark$ A target saturation of $94-98 \%$ is advised in most patients having PCA except those at risk of hypercapnic respiratory failure when a range of $88-92 \%$ should be achieved.

$\checkmark$ There is conflicting evidence concerning the balance of potential benefits and risks of perioperative hyperoxaemia to reduce the risk of surgical site infection in elective surgery and there is no evidence for this practice in patients having emergency surgical procedures. More trials are required for specific procedures, and more information is required concerning long-term mortality risks to patients with cancer. In the meantime, oxygen should not be used for this indication outside of clinical trials.

K. Oxygen use in palliative care (see full Guideline section 8.17)

$\mathrm{K} 1$. Oxygen use in palliative care patients should be restricted to patients with $\mathrm{SpO}_{2}$ consistently $<90 \%$ or patients who report significant relief of breathlessness from oxygen. In non-hypoxaemic patients, opioids and non-pharmacological measures should be tried before oxygen (grade $\mathrm{B}$ ).

K2. In general, there is no role for the monitoring of oxygen saturation or $\mathrm{PaO}_{2}$ in comfort-focused care in the last few days of life. If the patient appears comfortable, oxygen levels are irrelevant and should not influence care (grade D). 
Good practice points related to oxygen use in palliative care

Oxygen therapy for the symptomatic relief of breathlessness in palliative care patients is more complex than the simple correction of hypoxaemia. Consider the following issues:

$\checkmark$ Consider early involvement of palliative care specialists and physiotherapists.

$\checkmark$ As breathlessness is a multifactorial sensation-a comprehensive assessment of contributing factors (such as anxiety) should be carried out.

$\checkmark$ Low-dose opioids should be considered because they are effective for the relief of breathlessness in palliative care patients.

$\checkmark$ A trial of a handheld fan to help relieve breathlessness is recommended prior to trial of oxygen.

$\checkmark$ Oxygen use has to be tailored to the individual and a formal assessment made of its efficacy for reducing breathlessness and improving quality of life for that person.

$\checkmark$ Oxygen therapy should not be continued in the absence of patient benefit or where its disadvantages (eg, discomfort of masks or nasal cannulae, drying of mucous membranes) outweigh any likely symptomatic benefit.

L. Mixtures of oxygen with other gases (Heliox and Entonox)

Use of helium-oxygen mixtures (Heliox) (see full Guideline section 8.18):

L1. There is insufficient evidence to support the use of Heliox either as an inhaled gas or as the driving gas for nebuliser therapy in adult patients with acute exacerbations of asthma or acute exacerbations of COPD (AECOPD) except as part of randomised clinical trials or in exceptional circumstances (grade D).

L2. A therapeutic trial of Heliox is reasonable in patients with mechanical upper airway obstruction or postoperative stridor (grade D).

L3. Heliox use for patients with asthma or COPD should be considered only in clinical trials or in specialist hands for severe exacerbations that are not responding to standard treatment (and in COPD patients where there are contraindications to intubation) (grade D).

M. Use of nitrous oxide/oxygen mixtures (Entonox) for analgesia (see full Guideline section 9.11)

M1. The use of Entonox gas mixture for analgesia should be avoided if possible in patients at risk of hypercapnic respiratory failure (grade D).

N. CPAP and humidified high-flow nasal oxygen

Use of CPAP in the perioperative period and for pulmonary oedema (see online section 8.19):

N1. Patients with diagnosed sleep-disordered breathing established on CPAP undergoing surgery should bring their machines with them and use them in the preoperative and postoperative period. If adequate saturations are not achieved despite CPAP therapy, then assess for worsening ventilation with blood gases and oxygen should be entrained to achieve a saturation of 88-92\% (grade D).
N2. CPAP with entrained oxygen to maintain saturation 94-98\% should be considered to be an adjunctive treatment to improve gas exchange in patients with cardiogenic pulmonary oedema who are not responding to standard treatment in hospital care or in prehospital care (grade B).

Good practice point, high-flow humidified nasal oxygen via nasal cannulae

$\checkmark$ High flow nasal oxygen should be considered as a potentially superior alternative to reservoir mask treatment in patients with acute respiratory failure without hypercapnia. ${ }^{\text {ii }}$

P. Patients with tracheostomy or laryngectomy (see section 10.3)

$\mathrm{P} 1$. When oxygen is required by patients with prior tracheostomy or laryngectomy, a tracheostomy mask (varying the flow as necessary) should achieve the desired oxygen saturation (tables 1-4). An alternative delivery device, usually a T-piece device fitted directly to the tracheostomy tube, may be necessary if the patient deteriorates (grade D).

Q. Humidification of oxygen (see section 10.2)

Q1. Humidification is not required for the delivery of low-flow oxygen (mask or nasal cannulae) or for the short-term use of high-flow oxygen. It is not therefore required in prehospital care. Pending the results of clinical trials, it is reasonable to use humidified oxygen for patients who require high-flow oxygen systems for more than 24 hours or who report upper airway discomfort due to dryness (grade D).

Q2. In the emergency situation, humidified oxygen use can be confined to patients with tracheostomy or an artificial airway although these patients can be managed without humidification for short periods of time (eg, ambulance journeys) (grade D).

Q3. Humidification may also be of benefit to patients with viscous secretions causing difficulty with expectoration. This benefit can be achieved using nebulised normal saline (grade D).

Q4. Bubble bottles which allow a stream of oxygen to bubble through a container of water should not be used because there is no evidence of a clinically significant benefit but there is a risk of infection (grade $\mathrm{D})$.

Good practice points related to humidified oxygen therapy

$\checkmark$ Consider use of a large volume oxygen humidifier device for patients requiring high-flow rates or longer term oxygen, especially if sputum retention is a clinical problem.

$\checkmark$ In the absence of an artificial airway, the decision to humidify supplemental oxygen needs to be made on an individual basis but this practice is not evidence-based.

R. Driving gas for nebulised treatments (see section 10.4)

R1. For patients with asthma, nebulisers should be driven by piped oxygen or from an oxygen cylinder

${ }^{\mathrm{ii}}$ The prefix $\mathrm{O}$ is not in use for recommendations. 
fitted with a high-flow regulator capable of delivering a flow rate of $>6 \mathrm{~L} / \mathrm{min}$. The patient should be changed back to his/her usual oxygen mask or cannulae when nebuliser therapy is complete. If the cylinder does not produce this flow rate, an airdriven nebuliser (with electrical compressor) should be used with supplemental oxygen by nasal cannulae at $2-6 \mathrm{~L} / \mathrm{min}$ to maintain an appropriate oxygen saturation level (grade D).

R2. When nebulised bronchodilators are given to patients with hypercapnic acidosis, they should be given using an ultrasonic nebuliser or else a jet nebuliser driven by compressed air and, if necessary, supplementary oxygen should be given concurrently by nasal cannulae to maintain an oxygen saturation of $88-92 \%$. The same precautions should be applied to patients who are at risk of hypercapnic respiratory failure prior to the availability of blood gas results and the oxygen saturation should be monitored continuously during treatment. Once the nebulised treatment is completed for patients at risk of hypercapnic respiratory failure, their previous targeted oxygen therapy should be reinstituted (grade D).

\section{Good practice points}

Do not allow hypoxaemia to occur while administering nebulised treatments.

$\checkmark$ For hypoxaemic patients, oxygen therapy should continue during nebulised treatments.

Driving gas for nebulised treatment in ambulances:

$\checkmark$ During treatment by ambulance staff, oxygen-driven nebulisers should be used for patients with asthma and may be used for patients with COPD in the absence of an air-driven compressor system. If oxygen is used for patients with known COPD, its use should be limited to $6 \mathrm{~min}$. This will deliver most of the nebulised drug dose but limit the risk of hypercapnic respiratory failure (section 10.4). Ambulance services are encouraged to explore the feasibility of introducing battery powered, air-driven nebulisers or portable ultrasonic nebulisers.

S. Prescribing oxygen therapy (see full Guideline section 11)

S1. Every healthcare facility should have a standard oxygen prescription document or, preferably, a designated oxygen section on all drug prescribing cards or guided prescription of oxygen in electronic prescribing systems (grade D).

S2. A prescription for oxygen should always be provided, except in sudden illness when it must be started immediately and documented retrospectively (grade D).

S3. Doctors and other prescribers should prescribe oxygen using a target saturation range (sections 8,9 and 11) and sign the drug chart or electronic prescribing order (grade D).

S4. An oxygen target saturation range should be prescribed for all patients who are admitted to hospital. This will ensure that every patient will receive appropriate oxygen therapy if it should be required. It will also ensure that all clinicians are aware of the appropriate oxygen target range for every patient under their care (grade D).

Good practice points related to prescribing and administering oxygen therapy to patients

$\checkmark$ Oxygen should be prescribed on the drug chart or electronic prescribing system using a target saturation range.

$\checkmark$ Oxygen should be prescribed to a target saturation range rather than prescribing a fixed concentration of oxygen or fraction of inspired oxygen (see recommendations A1, A2, A4 and A5)

$\checkmark$ In most emergency situations, oxygen is given to patients immediately without a formal prescription. The lack of a prescription should never preclude oxygen being given when needed in an emergency situation. However, a subsequent written record must be made of what oxygen therapy has been given to every patient in a similar manner to the recording of all other emergency treatment.

$\checkmark$ If a patient has an oxygen alert card, initial oxygen therapy should be based on the guidance on the card until the results of blood gases are available.

T. Monitoring and adjusting oxygen therapy (see full Guideline sections 9-11)

T1. Pulse oximetry must be available in all locations where emergency oxygen is being used by healthcare professionals (see also the limitations of using pulse oximetry section 7.1.2) (grade D).

T2. All documents which record oximetry measurements or blood gas results should state whether the patient is breathing air or a specified oxygen delivery device and flow rate using the abbreviations shown in table 5 (grade D).

T3. In all situations where repeated blood gas measurements are required, they should be measured as soon as possible, usually within $30 \mathrm{~min}$ of any treatment change, to determine if the proposed target saturations are appropriate. Consider the use of an indwelling arterial catheter if multiple samples are likely to be required (grade D).

T4. Adjustments should only be made by registered staff who have been trained to administer oxygen. If the oxygen saturation falls below the prespecified range, the concentration of oxygen should be increased; if the saturation rises above this range, the oxygen concentration should be reduced. If the monitoring of oxygen saturation is performed by unregistered staff (eg, healthcare assistants), there must be a clear protocol in place which requires that they should inform staff who are trained to administer oxygen if the oxygen saturation is above or below the target saturation (grade $\mathrm{D}$ ).

Good practice points related to administration of oxygen therapy

$\checkmark$ For hypoxaemic patients, oxygen therapy should continue during other treatments such as nebulised therapy. Clinicians should assess the clinical status of the patient prior to prescribing oxygen and the patient's condition should be reassessed frequently during oxygen use (see recommendations B1-B3). 
$\checkmark$ The administering healthcare professional should note the oxygen saturation before starting oxygen therapy wherever possible but never discontinue or delay oxygen therapy for seriously ill patients (see recommendation $\mathrm{B} 2$ ).

$\checkmark$ The healthcare professional should start oxygen therapy using an appropriate delivery system and flow rate as specified in sections $8-10$ of this guideline. The target oxygen saturation should be documented on the respiratory section of the observation chart.

$\checkmark$ Whenever possible, patients should be given an oxygen information sheet (example in web appendix 6 on the BTS website).

$\checkmark$ Staff should check the oxygen supply and connections on a regular basis because there have been serious incidents due to disconnection or misconnection of oxygen supplies.

$\checkmark$ Staff must ensure that adequate oxygen is provided during transfers and while patients are in diagnostic departments. Additionally, oxygen saturation should be monitored continuously for seriously ill patients who require escorted transfers. This is because there have been serious incidents involving accidental discontinuation of oxygen or cylinders running out during interward transfers or transfers to other departments such as for X-rays.

$U$. Weaning and discontinuation of oxygen therapy

U1. Lower the oxygen concentration if the patient is clinically stable and the oxygen saturation is above the target range or if it has been in the upper zone of the target range for some time (usually 48 hours) (grade $\mathrm{D}$ ).

U2. If the target saturation is maintained, the new delivery system and flow should be continued. Repeat blood gas measurements are not required. If the patient is stable, the process can be repeated and the patient can eventually be weaned off oxygen (see section 12) (grade D).

U3. Most stable convalescent patients will eventually be stepped down to $2 \mathrm{~L} / \mathrm{min}$ via nasal cannulae prior to cessation of oxygen therapy. Patients at risk of hypercapnic respiratory failure may be stepped down to $1 \mathrm{~L} / \mathrm{min}$ (or occasionally $0.5 \mathrm{~L} / \mathrm{min}$ ) via nasal cannulae or a $24 \%$ Venturi mask at $2 \mathrm{~L} / \mathrm{min}$ as the lowest oxygen concentration prior to cessation of oxygen therapy (grade D).

U4. Oxygen therapy should be stopped once a patient is clinically stable on low-concentration oxygen, and the oxygen saturation is within the desired range on two consecutive observations (but the prescription for a target saturation range should remain active in case of future deterioration). It may be appropriate to alter the target range following senior review in patients with chronic cardiopulmonary disease who either have saturations $<94 \%$ when stable or in whom it is deemed sensible to discharge from hospital with saturations $<94 \%$ pending an outpatient oxygen assessment. Oxygen should also be stopped if the patient has come to the end of a written protocol of timed oxygen (eg, postoperatively) (grade D).

U5. Oxygen saturation on air should be monitored for 5 min after stopping oxygen therapy. If it remains in the desired range, it should be rechecked at 1 hour (grade D).

U6. If the oxygen saturation and physiological 'track and trigger' score (eg, NEWS) is satisfactory at 1 hour, the patient has safely discontinued oxygen therapy. However, saturation and physiology should continue to be monitored on a regular basis according to the patient's underlying clinical condition (grade D).

U7. If the saturation falls below the patient's target range on stopping oxygen therapy, restart the lowest concentration that maintained the patient in the target range and monitor for $5 \mathrm{~min}$. If this restores the saturation into the target range, continue oxygen therapy at this level and attempt discontinuation of oxygen therapy again at a later date provided the patient remains clinically stable (grade $\mathrm{D}$ ).

U8. If a patient requires oxygen therapy to be restarted at a higher concentration than before to maintain the same target saturation range, the patient should have a clinical review to establish the cause for this deterioration (grade D).

U9. Some patients may have episodic hypoxaemia (eg, after minor exertion or due to mucus plugging) after they have safely discontinued oxygen therapy. An on-going prescription for a target saturation range will allow these patients to receive oxygen as the need arises but transient asymptomatic desaturation does not require correction (grade D).

V. Practical aspects of oxygen use in prehospital and hospital care and use of oxygen alert cards (see full Guideline sections 9-11)

V1. Emergency oxygen should be available in primary care medical centres, preferably using oxygen cylinders with integral high-flow regulators. Alternatively, oxygen cylinders fitted with high-flow regulators (delivering up to $15 \mathrm{~L} / \mathrm{min}$ ) must be used to allow use with reservoir masks (grade $\mathrm{D}$ ).

V2. Healthcare organisations should take measures to eliminate the risk of oxygen tubing being connected to the incorrect wall oxygen outlet or to outlets that deliver compressed air or other gases instead of oxygen. Air flow metres should be removed from the wall sockets or covered with a designated air outlet cover when not in use. Special care should be taken if twin oxygen outlets are in use (grade D).

Good practice points related to practical aspects of oxygen therapy

Assessment and immediate oxygen therapy:

$\checkmark$ Chronically hypoxaemic patients with a clinical exacerbation associated with a $3 \%$ or greater fall in oxygen saturation on their usual oxygen therapy 
should usually be assessed in hospital with blood gas estimations. $\mathrm{PaO}_{2}$ of $<7 \mathrm{kPa}$ equates to $\mathrm{SpO}_{2}$ below $\sim 85 \%$.

$\checkmark$ The initial oxygen therapy to be used in the various clinical situations is given in tables 1-4.

$\checkmark$ If there is a clear history of asthma or heart failure or other treatable illness, appropriate treatment should be instituted in accordance with guidelines or standard management plans for each disease.

$\checkmark$ The oxygen saturation should be monitored continuously until the patient is stable or arrives at hospital for a full assessment. The oxygen concentration should be adjusted upwards or downwards to maintain the target saturation range.

$\checkmark$ In most emergency situations, oxygen is given to patients immediately without a formal prescription or drug order. The lack of a prescription should never preclude oxygen being given when needed in an emergency situation. However, a subsequent written record must be made of what oxygen therapy has been given to every patient (in a similar manner to the recording of all other emergency treatment).

$\checkmark$ General practitioners or first responders visiting a patient's home should carry a portable pulse oximeter to assess hypoxaemia and guide use of oxygen if available and should call emergency services if hypoxaemia or other serious illness is suspected.

$\checkmark$ Those attending patients as an emergency in rural or remote areas should consider carrying a portable oxygen cylinder as part of their emergency equipment.

Oxygen alert cards for patients with hypercapnic respiratory failure:

$\checkmark$ Patients with COPD (and other at-risk conditions) who have had an episode of hypercapnic respiratory failure should be issued with an oxygen alert card and with a $24 \%$ or $28 \%$ Venturi mask. They should be instructed to show the card to the ambulance crew and emergency department staff in the event of an exacerbation.

$\checkmark$ Oxygen alert cards with agreed content can be obtained via the BTS website.

$\checkmark$ The content of the alert card should be specified by the physician in charge of the patient's care, based on previous blood gas results.

$\checkmark$ The primary care team and ambulance service should also be informed by the hospital COPD team that the patient has had an episode of hypercapnic respiratory failure and carries an oxygen alert card. The home address and ideal oxygen concentration or target saturation ranges of these patients can be flagged in the ambulance control systems and information disseminated to ambulance crews when required.

$\checkmark$ When possible, out-of-hours services providing emergency primary care services should be informed by the hospital COPD team or by the primary care team that the patient has had an episode of hypercapnic respiratory failure and carries an oxygen alert card. Use of oxygen in these patients will be guided by the instructions on the alert card or by a Patient Specific Protocol which can be shared by hospital teams, the ambulance service and the primary care team.

W. Practical aspects of oxygen dispensing, documentation and monitoring

W1. Registered nurses and others who dispense drugs in hospitals should sign the drug chart or electronic prescribing record at every drug round and check that the patient is receiving oxygen therapy. This is to check that the patient is within the target saturation and also to check whether weaning and discontinuation should be instituted (grade D).

W2. Most patients are prescribed an oxygen target range. If patients are on air at the time of the drug round, registered nurses should sign the drug chart using a code such as 'A' for air and the observation chart should also be filled in using the code A for air (see table 5 and figure 19 in the full guideline) (grade D).

W3. All patients should have their oxygen saturation observed for at least 5 min after starting oxygen therapy or for patients who require an increased concentration of oxygen and after oxygen therapy has been decreased or stopped (grade D).

W4. If the oxygen saturation is above the target saturation range and the patient is stable, the delivery system or oxygen flow rate should be modified to return the saturation to within the target range (grade D).

W5. Patients who have a target saturation of $88-92 \%$ should have their blood gases measured within $30-60 \mathrm{~min}$. This is to ensure that the carbon dioxide level is not rising. This recommendation also applies to those who are at risk of developing hypercapnic respiratory failure but who have a normal $\mathrm{PCO}_{2}$ on the initial blood gas measurement (grade D).

W6. Stable patients whose oxygen saturation is within their target saturation range of $94-98 \%$ do not need repeat blood gas measurements within 30-60 min if there is no risk of hypercapnic respiratory failure and acidosis and may not need any further blood gas measurements unless there should be further deterioration including symptoms or signs of possible hypercapnia (grade D).

W7. Stable patients on oxygen treatment should have $\mathrm{SpO}_{2}$ and physiological variables (eg, NEWS) measured four times a day (grade D).

W8. In those who have signs of critical illness (eg, NEWS score 7 or above), oxygen saturation should be monitored continuously and the patient may require level 2 or level 3 care on a highdependency unit or critical care unit (grade D).

W9. If the patient is clinically stable and the oxygen saturation is within the target range, treatment should be continued (or eventually lowered) depending on the clinical situation (grade D). 
W10. Oxygen therapy should be increased if the saturation is below the desired range and decreased if the saturation is above the desired range (and eventually discontinued as the patient recovers) (grade D).

W11. The new saturation (and the new delivery system) and flow rate should be recorded on the patient's observation chart after 5 min of treatment at the new oxygen concentration. Each change should be recorded by the clinician trained to administer oxygen by signing the observation chart (only changes should be signed for) (grade D).

W12. Repeat blood gas measurements are not required for stable patients who require a reduced concentration of oxygen (or cessation of oxygen therapy) to maintain the desired target saturation (grade D).

W13. Patients with no risk of hypercapnic respiratory failure do not always need repeat blood gas measurements after an increase in oxygen concentration. However, the patient requires clinical review to determine why the oxygen saturation has fallen (grade D).

W14. Patients at risk of hypercapnic respiratory failure (usually those with a target range of $88-92 \%$; see table 4) require repeat blood gas assessment 30$60 \mathrm{~min}$ after an increase in oxygen therapy (to ensure that the carbon dioxide level is not rising) (grade D).

W15. For patients with no risk of hypercapnic respiratory failure, monitoring by pulse oximeter is sufficient (repeated blood gases not required) provided the patient is clinically stable and the oxygen saturation remains in the desired range, usually $94-98 \%$ (grade D).

W16. If a patient's oxygen saturation is lower than the prescribed target range, first check all aspects of the oxygen delivery system and the oximeter device for faults or errors (grade D).

W17. If a patient's oxygen saturation is consistently lower than the prescribed target range, there should be a medical review and the oxygen therapy should be increased according to an agreed written protocol (grade D).

W18. If the oxygen saturation fails to rise following 5-10 min of increased oxygen therapy or if there is clinical concern following medical review, then blood gas measurements should be repeated (grade D).

$X$. Training in oxygen prescribing and use

X1. All clinicians prescribing oxygen should have appropriate training and access to written or electronic oxygen prescribing guidelines based on this national guideline (grade D) (see online appendices 7 and 8).

X2. Every hospital should have a training programme to ensure that clinical staff are familiar with the hospital's oxygen administration policies. In view of the high number of adverse incidents related to oxygen therapy, it is recommended that all acute trusts should include basic training in oxygen use in the mandatory training programmes for all clinical staff (grade D).

Healthcare providers need to use clinical judgement, knowledge and expertise when deciding whether it is appropriate to apply recommendations for the management of patients. The recommendations cited here are a guide and may not be appropriate for use in all situations. The guidance provided does not override the responsibility of healthcare professionals to make decisions appropriate to the circumstances of each patient, in consultation with the patient and/or their guardian or carer.

Collaborators BTS Emergency Oxygen Guideline Group: BROD, LH, JE, VM, Dr Sabrina Bajwah, Professor Richard Beasley, Dr Katrina Curtis, Professor Anthony Davison, Dr Alistair Dorward, Dr Chris Dyer, Angela Evans, Lucy Falconer, Clare Fitzpatrick, Dr Simon Gibbs, Dr Kim Hinshaw, Dr Robin Howard, Dr Binita Kane, Dr Jeff Keep, Carol Kelly, Hasanin Khachi, Dr Muhammed Asad Iqbal Khan, Dr Roop Kishen, Leigh Mansfield, Dr Bruce Martin, Dr Fionna Moore, Dr Duncan Powrie, Dr Louise Restrick, Professor Christine Roffe, Dr Mervyn Singer, Dr Jasmeet Soar, Dr lain Small, Lisa Ward, David Whitmore, Professor Wisia Wedzicha, Dr Meme Wijesinghe.

Contributors BROD was lead author responsible for the overall document and production of supporting appendices. LH was lead author for sections 3-6. JE was lead author for section 14. VM was lead author for sections 9-11. All authors were responsible for the final approval of the guideline. Members of the guideline group and their contributions to individual sections of the guideline are listed in Annex 1 of the full guideline.

Competing interests $\mathrm{LH}$ has received research funding support from Bayer PLC. VM has received lecture and meeting attendance support from Chiesi, GSK and AstraZeneca.

Provenance and peer review Not commissioned; internally peer reviewed.

Data sharing statement No additional data are available.

\section{ONLINE APPENDICES-AVAILABLE AT http://www.brit- thoracic.org.uk}

1. Search strategy

2. Evidence tables

3. Summary of guideline for hospital use

4. Example of local oxygen policy

5. Summary of guideline and flow charts for emergency oxygen use in ambulances, community and prehospital settings

6. Patient information sheet

7. Slides on emergency oxygen use for doctors

8. Slides on emergency oxygen use for nurses, midwives, pharmacists, physiotherapists and other practitioners who use oxygen

9. Key points for hospital managers and oxygen champions

10. Key points for primary care managers, clinical commissioning groups

11. Dissemination and implementation of the guideline

\section{REFERENCES}

1. O'Driscoll BR, Howard LS, et al. BTS Guideline for oxygen use in adults in healthcare and emergency settings, Thorax 2017;72 (Suppl 1):pi1-i89.

2. O'Driscoll BR, Howard LS, Davison AG, et al. BTS guideline for emergency oxygen use in adult patients. Thorax 2008;63(Suppl 6): vi1-68.

3. Hardinge M, Annandale J, Bourne S, et al. British Thoracic Society guidelines for home oxygen use in adults. Thorax 2015;70(Suppl 1): i1-43. 
4. Hale KE, Gavin C, O'Driscoll BR. Audit of oxygen use in emergency ambulances and in a hospital emergency department. Emerg Med J 2008;25:773-6.

5. Wilson AT, Channer KS. Hypoxaemia and supplemental oxygen therapy in the first 24 hours after myocardial infarction: the role of pulse oximetry. J R Coll Physicians Lond 1997;31:657-61.

6. Cook DJ, Reeve BK, Griffith LE, et al. Multidisciplinary education for oxygen prescription. A continuous quality improvement study. Arch Intern Med 1996;156:1797-801.

7. Small D, Duha A, Wieskopf B, et al. Uses and misuses of oxygen in hospitalized patients. Am J Med 1992;92:591-5.

8. Dodd ME, Kellet F, Davis A, et al. Audit of oxygen prescribing before and after the introduction of a prescription chart. BMJ 2000;321:864-5.

9. Howell M. An audit of oxygen prescribing in acute general medical wards. Prof Nurse 2001;17:221-4.
10. Boyle M, Wong J. Prescribing oxygen therapy. An audit of oxygen prescribing practices on medical wards at North Shore Hospital, Auckland, New Zealand. N Z Med J 2006;119: U2080.

11. Kbar FA, Campbell IA. Oxygen therapy in hospitalized patients: the impact of local guidelines. J Eval Clin Pract 2006;12:31-6.

12. AGREE II Instrument. Secondary AGREE II Instrument. http://www. agreetrust.org/agree-ii/.

13. JRCALC. UK Ambulance Services Clinical Practice Guidelines 2013. London: JRCALC, 2013.

14. BTS. British Thoracic Society Guideline Production Manual. London: BTS, 2014:31.

15. COPD NICE Clinical Guideline 101, 2010

16. Davidson AC, et al. BTS/ICS guideline for the ventilatory management of acute hypercapnic respiratory failure in adults. Thorax 2016;(Suppl 2) 71ii1-ii35. 